\title{
Hacia una crítica de las prácticas de enseñanza de la filosofía en Colombia: aproximaciones desde Michel Foucault
}

Diego Fernando Camelo Perdomo

Universidad de San Buenaventura, Colombia 


\title{
Hacia una crítica de las prácticas de enseñanza de la filosofía en Colombia: aproximaciones desde Michel Foucault*
}

Resumen: en este artículo se procura presentar, desde algunos postulados del filósofo francés Michel Foucault, una crítica a los elementos que componen el conjunto de prácticas de enseñanza de la filosofía a nivel de bachillerato en Colombia, donde la función de algunas instituciones se encuentra enmarcada en la vigilancia del saber mediante la implementación de prácticas examinadoras de su enseñanza y su aprendizaje. No obstante, en este proceso dinámico se han presentado una serie de cambios discursivos que provocaron una discontinuidad en el ejercicio didáctico de este saber.

Palabras clave: enseñanza, filosofía, dispositivo disciplinario, prácticas examinadoras, Foucault.

\section{Towards a critic of the philosophy teaching practices in Colombia: approaches from Michel Foucault}

\begin{abstract}
French philosopher Michel Foucault. It is a critic of the elements that compose the philosophy teaching practices in Colombian High Schools. Here the function of some institutions is framed in the surveillance of the knowledge through examination practices of philosophy teaching and learning. However, in this dynamic process, a series of discursive changes have appeared that caused discontinuity in the didactic exercise of this knowledge.
\end{abstract}

Keywords: teaching, philosophy, disciplinary dispositive, examination practices, Foucault.

Fecha de recepción: 2 de octubre de 2019

Fecha de aceptación: 10 de marzo de 2020

Forma de citar (APA): Camelo-Perdomo, D. (2020). Hacia una crítica de las prácticas de enseñanza de la filosofía en Colombia: aproximaciones desde Michel Foucault. Revista Filosofía UIS, 19(2). https://doi.org/10.18273/revfil.v19n2-2020014

Forma de citar (Harvard): Camelo-Perdomo, D. (2020). Hacia una crítica de las prácticas de enseñanza de la filosofía en Colombia: aproximaciones desde Michel Foucault. Revista Filosofía UIS, 19(2), 263-283.

Diego Fernando Camelo Perdomo: colombiano. Licenciado en Filosofía por la Universidad Santo Tomás, Bogotá. Maestrante en Filosofía Contemporánea. Universidad de San Buenaventura, Colombia.

ORCID iD: orcid.org/0000-0003-3068-1997

Correo electrónico: diego.camelo.p@gmail.com; dfcamelop@academia.usbbog.edu.co

*Artículo de reflexión derivado de investigación. 


\section{Hacia una crítica de las prácticas de enseñanza de la filosofía en Colombia: aproximaciones desde Michel Foucault ${ }^{1}$}

\section{Introducción}

Se considera que si en algo no se debe caer durante la lectura de este artículo es en el supuesto "antididacticismo filosófico" en el que algunos críticos suelen sucumbir, como si criticar significara simplemente adoptar una actitud negativa frente a algo con la pretensión de rechazo y ya; no, eso no es crítica. Cuando en este texto se habla de crítica se toma en el sentido foucaultiano en el que, a su vez, intrínsecamente se halla la influencia de Kant. En este orden de ideas, criticar las prácticas de la enseñanza de la filosofía no se limita a la agonía discursiva sobre lo que pudo haber sido y no fue. La crítica consistirá en analizar cuáles serían las condiciones de posibilidad de dichas prácticas para resaltar sus límites y alcances conceptuales, sin obviar las circunstancias históricas en las que emergen. Por esta razón, se desarrolla en tres puntos los componentes de la crítica que se realiza; los cuales, para la comprensión, se mencionan a continuación.

Primero se realizará una aproximación a la noción de crítica desarrollada por Michel Foucault, a partir de un recorrido por algunas de sus obras, desde Las palabras y las cosas (1967) hasta su reconocida conferencia pronunciada en 1978 ante la Sociedad francesa de filosofía titulada ¿Qué es la crítica? Luego, se intentará interpretar la función del Instituto Colombiano para la Evaluación de la Educación (Icfes en adelante) como un dispositivo disciplinario de la filosofía en Colombia el cual está conformado por ciertas prácticas tales como la evaluación y la enseñanza de los temas curriculares. No obstante, hay una tecnología que opera en este dispositivo de poder, pues se trata más bien un modo en que funciona la práctica de la enseñanza en este entramado: la educación por competencias, y este es, precisamente, el tercer punto a desarrollar.

${ }^{1}$ Este artículo hace parte de los resultados analizados en una investigación que el autor realizó sobre las prácticas didácticas de la filosofía a nivel de bachillerato en Colombia. Una versión previa fue presentada en el VI Congreso Colombiano de Filosofía llevado a cabo en la Universidad del Norte de la ciudad de Barranquilla (Atlántico) entre los días 13 al 16 de agosto de 2016 y organizado por la Sociedad Colombiana de Filosofía. 


\section{Aproximación a la noción de Crítica en M. Foucault}

Quizás uno de los conceptos más característicos en el pensamiento de Michel Foucault es la crítica. No obstante, es menester delinear el alcance de su empleo dentro de lo que para él la actividad filosófica se traducía como trabajo crítico. Por esta razón se tiene como carta de navegación la obra Sobre la Ilustración, en la cual están contenidas tres elaboraciones de pilar importancia en lo que respecta a la consideración sobre la crítica. Estas son: ¿Qué es la crítica? (Crítica y Aufklärung)2 ${ }^{2}$, Seminario sobre el texto de Kant "Was ist Aufklärung" y ¿Qué es la ilustración?

El indudable punto de partida de la disertación sobre la crítica hecha por Foucault es Kant, pero con algunas connotaciones que singularizan esta definición. Él sostiene que alrededor de la crítica ha existido una cierta manera de pensar, de decir, pero también de actuar; una relación entre lo que existe, se sabe y se hace; una relación entre la sociedad y la cultura. A todo lo anterior el pensador francés lo llamó actitud crítica (Foucault, 2003, p. 4). Por consiguiente, la crítica existe en orden a la relación instrumental con que se pretende medir y verificar. En últimas, la crítica tendría una función subordinada en relación a aquello que se pretende enfrentar, que hace que la crítica se asimile como una actitud imperativa, por lo que el autor definió la crítica como una virtud (Foucault, 2003, p. 5).

Foucault cree que esta definición obedece al ejercicio de la pastoral cristiana, cuyas prácticas de individualización influyeron —como el arrepentimiento, la penitencia, la oración y la confesión - en que los individuos pensaran que debían ser gobernados y dejarse gobernar, es decir, encausaran su conducta a la salvación. Sin embargo, el meollo del problema no consiste en cómo gobernar, sino en cómo no ser gobernado, pero esta negación sería sobre los modos de ser. Entonces, saber cuáles son los modos y los medios de gobierno, es decir, las tecnologías de gubernamentalidad fue lo que Foucault llamó actitud crítica. A partir de esta idea, el pensador hace mención de dos puntos de referencia: el primero es que la crítica obliga a un tipo de rechazo a los modos de ser gobernados; una limitación a las practicas individualizantes. El segundo es que la crítica es una oposición, es decir, al momento de rechazar el modo de ser gobernado, a su vez, es una oposición a las leyes que legitiman la gubernamentalidad. Finalmente, no querer ser gobernado implica no aceptar como verdadera la autoridad que se impone. Cada una de estas salvedades representa un elemento que constituye la relación y en cuyo foco surge la crítica. Los elementos a los que se hace referencia son el poder, el sujeto y la verdad.

\footnotetext{
${ }^{2}$ Este texto corresponde a una conferencia expuesta ante la Sociedad Francesa de Filosofía en la sesión del 27 de mayo de 1978. Para efectos del presente trabajo se tendrá como fuente bibliográfica una versión reciente que obedece a la traducción de De la Higuera, Bello \& Campillo (2003).
} 

en Colombia: aproximaciones desde Michel Foucault

\begin{abstract}
Yo diría que la crítica es el movimiento por el cual el sujeto se atribuye el derecho de interrogar a la verdad acerca de sus efectos de poder $y$ al poder acerca de sus discursos de verdad; la crítica será el arte de la incertidumbre voluntaria, de la indocilidad reflexiva. La crítica tendría esencialmente como función la desujeción en el juego de lo que se podría dominar, con una palabra, la política de a verdad. (Foucault, 2003, p. 11)
\end{abstract}

Según lo dicho por el autor, la definición de crítica se asemeja a la que Kant hizo de la ilustración. De hecho, se pregunta sobre la manera de situar su concepto de crítica con el de la ilustración de Kant. A tenor de esta consideración, en la obra Una lectura de Kant (2009), Foucault analiza la inserción del concepto de antropología con lo presentado en la Crítica de la razón pura (1781), esto resulta en la identificación de una relación de finalidad, pues tanto en la Crítica como en Antropología en sentido pragmático se descubre "una cierta verdad crítica del hombre hija de la crítica de las condiciones de verdad" (Foucault, 2009, p. 41). Esto permite pensar que la crítica surge de las condiciones de posibilidad de la verdad.

Ahora bien, la caracterización que Foucault hace de la crítica resalta tres rasgos de la crítica kantiana en relación a la Aufklärung. Primero, era una ciencia que inspiraba confianza en sí misma; segundo, el desarrollo de un Estado que se mostraba a sí mismo como racionalidad histórica; y tercero, era una ciencia del estado o un estatalismo (Foucault, 2003, p. 15). Entonces, todo esto se encamina a explicar cuál es el significado de la pregunta ¿qué es la Aufklärung?, por lo que se responde con la confirmación de que esta pregunta no está dirigida a entender la Ilustración como una época; por el contrario, el texto de Kant es para Foucault una cuestión del ahora.

La cuestión que a mi juicio surge por primera vez en este texto de Kant es la cuestión del presente, la cuestión de la actualidad: ¿qué es lo que ocurre hoy?, ¿qué es lo que pasa ahora?, ¿qué es ese "ahora" en el interior el cual estamos unos y otros y que define el momento en el escribo? (Foucault, 1985, p. 198)

Esto mismo parece ocurrirle a Foucault cuando aborda la cuestión de la modernidad, cuyo discurso contiene inmerso una cuota de actualidad donde pueda develar el sentido y así especificar el modo de acción dentro de ella: “¿Cuál es mi actualidad? ¿Cuál es el sentido de esta actualidad? ¿Qué es lo que hago cuando hablo de ella? He aquí la singularidad de esta nueva interrogación acerca de la modernidad" (Foucault, 1985, p. 200). Por consiguiente, para Foucault la pregunta ¿Qué es la Aufklärung? significa una cuestión que rodea los excesos de poder, los procesos de gubernamentalización y la responsabilidad histórica de la razón en la que se basan sus prácticas. 
En esta dirección, el despliegue de la cuestión crítica se ha visto en cierta medida condicionada por las estructuras de poder político ${ }^{3}$ y la ha condenado a un sinsentido. Por eso es que Foucault (2003) se pregunta: "¿cómo puede ser que haya sentido a partir del sinsentido?" (p. 18). Es por eso que el problema del sentido es un asunto de racionalidades que tienen efectos de poder $y$, en consecuencia, el problema de la crítica es una cuestión por el sentido, entendiéndolo como una creación de las estructuras de poder o maquinarias significantes, en palabras del autor.

La relación entre la cuestión del sentido y la institucionalización se vigoriza en una práctica que Foucault llamó histórico-filosófica, la cual consiste en construir una historia conformada por las relaciones entre estructuras racionales que articulan un discurso y un mecanismo de sujeción (Foucault, 2003, p. 21). Con ello se demuestra que el objeto de la historia de los historiadores queda desplazado por el problema del sujeto y la verdad, lo que significa la emancipación o la liberación de la historia al centrar el interés sobre los efectos del poder que afectan la verdad de la que depende. Por esto es que la práctica histórico-filosófica, en el caso de Kant y la Aufklärung por ejemplo, no tiene un periodo predeterminado, pues es aplicable a cualquier tiempo y lugar debido a que el trabajo de esta práctica radica en analizar las relaciones de poder, verdad y sujeto. En esta dirección, la crítica no busca cuestionar los absolutismos históricos sino identificar las condiciones que hacen posibles las relaciones entre los tres elementos que se han sido mencionados en líneas atrás. Para el caso, se tiene la siguiente cita:

Lo que hace que tal procedimiento de coerción adquiera la forma y las justificaciones. Lo que buscamos, entonces, no es saber lo que es verdadero o falso, fundado o no fundado, real o ilusorio, científico o ideológico, legítimo o abusivo. Buscamos saber cuáles son los lazos, las conexiones que pueden ser señaladas entre mecanismos de coerción y elementos de conocimiento, qué juegos de reenvío y de apoyo se desarrollan entre unos y otros, qué hace que tal elemento de conocimiento pueda tomar unos efectos de poder referidos, en un sistema tal, a un elemento verdadero o probable, incierto o falso, y propias de un elemento racional, calculado, técnicamente eficaz, etc. (Foucault, 2003, p. 26)

Verdad y poder son un binomio que dentro del discurso de la crítica cumplen roles convergentes y complementarios. El primero se refiere a los procedimientos y a todos los efectos de conocimientos que son aceptables en un momento dado. Por su parte, el segundo término tiene la función de cobijar los mecanismos que están definidos pero que a la vez definen comportamientos y discursos (Foucault, 2003, p. 27). Este binomio tiene también un papel metodológico. Es como una

\footnotetext{
${ }^{3}$ Foucault hace mención de los casos de Alemania y Francia donde el problema de la Aufklärung es abordado con marcadas diferencias políticas y epistemológicas.
} 
rejilla de análisis a través de la cual se fijan los elementos que deben ser parte de las condiciones de posibilidad.

Los mecanismos de poder despliegan su funcionamiento de acuerdo a los instrumentos, los medios o procedimientos que validen la coherencia de un sistema. Con base en esto se puede afirmar que la crítica tiene una función arqueológica debido a que no se trata de describir qué es el poder ni qué es el saber, pues de ser así se estarían tomando como absolutos, sino más bien de describir las relaciones entre saber y poder que permitan identificar la aceptabilidad de un sistema determinado. No obstante, Foucault cree que la función arqueológica de la crítica lleva consigo tres consecuencias que vale la pena mencionarlas brevemente. La primera es que la aceptabilidad de un sistema no insinúa ninguna evidencia anterior a éste: no hay ningún a priori que lo anteceda. La segunda es que el conjunto que conforma el sistema no se analiza como un universal como lo pretende la historia. Cuando de lo que se trata es que se aplique este análisis como singularidades, ellas serían la garantía de que el método histórico-filosófico no sea simplemente historia. Y la tercera, el análisis de las singularidades no centra su atención a reconstruir un conjunto de fenómenos nacidos por una causa, sino en hacer una reconstrucción de las condiciones en que aparecieron estas singularidades en medio de la multiplicidad. Esta última consecuencia hace viable que la crítica tenga también una función genealógica, en cuanto que abre las posibilidades de relaciones inteligibles, a modo de juego de interacción, donde se ponen en movimiento intenciones, reglas y acciones singulares cuya organización está dada de forma estratégica.

Así, en la crítica convergen tres funciones, a saber: la función arqueológica, la función genealógica y la función estratégica. A partir de lo anterior Foucault plantea el problema de la crítica, es decir que la formulación de esta cuestión sobrepasa los términos de conocimiento y legitimación, y prefiere abordarlos desde el poder y la eventualización, pero con una salvedad, y es que el poder no seguir entendiéndose como un mero ejercicio de dominación y opresión, sino más bien como relación en un espacio de interacción donde se involucran formas de saber. En últimas no se trata de preguntarse por el origen del poder, antes bien, se trata de cuestionar por las relaciones circunstanciales de su emergencia.

\section{El Icfes como dispositivo disciplinario de la filosofía}

Cuando se habla de dispositivo se alude a un entramado de relaciones de prácticas, tanto discursivas como no discursivas, cuyas técnicas las singularizan dentro de esa relación. Cada práctica no se define por ser ella en sí misma, sino por la función que ejerce dentro del dispositivo. El propósito de este punto, que conforma la crítica de la práctica de la enseñanza de la filosofía en Colombia, 
es descifrar el horizonte de posibilidad que tiene el Icfes en tanto dispositivo de disciplina, ya no de los cuerpos, sino de los saberes, es decir, se habla de un disciplinamiento de los saberes con efectos somáticos.

Por consiguiente, se realiza un breve despliegue sobre qué se entiende, a la luz del pensamiento de Foucault, por saber disciplinario y disciplinamiento de los saberes, aspectos que son abordados en su clase del 25 de febrero de 1976 correspondientes al curso Defender la sociedad. Esta relación — saber disciplinario y disciplinamiento de los saberes - surge a partir de la distinción que se logra establecer entre la historia de las ciencias y la genealogía de los saberes, distinción que Foucault (2000) caracteriza de la siguiente manera: la primera se ubica dentro de la relación binaria conocimiento-verdad. Por su parte, la segunda lo hace dentro de la dinámica entre discurso-poder (p. 167). Y, este último es el encargado de superar la tradicional pugna en el siglo XVIII (Ilustración) entre el conocimiento y la ignorancia. En esta dirección, el problema radica, según Foucault, no en el enfrentamiento entre quién sabía y quién no, sino, más bien, entre los mismos saberes. La multiplicidad de saberes hizo que los rasgos diferenciales de cada uno de ellos se situaran en un escenario de lucha donde lo único que quedó fue la fragmentación entre ellos.

Para ambientar y ejemplificar de forma clara este fenómeno, Foucault recurre a un tipo de resignificación del siglo XVIII y al surgimiento del saber técnico, el cual nace no del enfrentamiento entre la ignorancia y el conocimiento, sino de la interacción entre la producción y las demandas económicas que provocó que el saber fuera más mezquino tanto en su acceso como en su ejercicio. Con base en esto se deduce que debido al dinamismo económico — producción-demandael saber técnico se catapulta a una esfera de poder. En ese sentido, quien poseía el saber, ejercía el poder. Ahora bien, como el saber representaba poder, el Estado no podía permitir que de forma paralela a él se ejerciera poder. Por este motivo, el Estado tuvo intervino en la lucha de saberes al implementar, según lo propone Foucault (2000), cuatro procedimientos, a saber: primero, la eliminación de los saberes inútiles y costosos; segundo, la normalización de los saberes entre sí al generar relaciones de poder; tercero, la jerarquización de los saberes que permite encajarlos unos con otros; y cuarto, la centralización que controla esos saberes, tanto en su transmisión como en su direccionamiento (p. 198).

Por consiguiente, la intervención del Estado en esta lucha de saberes es algo que Foucault Ilamó la homogenización de los saberes tecnológicos. El poder del Estado cumple una función homogeneizadora, a partir de la cual surge la necesidad de crear Escuelas Superiores que permitieran establecer niveles cualitativos y cuantitativos de los saberes y posibilitó la jerarquización de los mismos. Una vez jerarquizados los saberes, entra en escena el cuerpo de 
inspectores, es decir, agentes cuyo encargo es indicar el aprovechamiento de los saberes técnicos. De esta manera, la utilidad de los saberes queda incrustada en prácticas normalizadoras dispuestas por quienes centralizan sus funciones. De esta manera, es como el Estado ejerce sobre el saber lo que Foucault llamó el poder disciplinario. Foucault (2000) sostiene que,

El siglo XVIII fue el siglo del disciplinamiento de los saberes, es decir, la organización interna de cada uno de ellos como una disciplina que tiene, en su campo de pertenencia, a la vez criterios de selección que permiten desechar el falso saber, el no saber, formas de normalización y de homogenización de los contenidos, formas de jerarquización y, por último, una organización interna de centralización de lesos saberes en torno de una especie de axiomatización de hecho. (p. 170)

Al ser ordenado cada saber en cuanto disciplina, se produce una jerarquización global a la que se denominó ciencia (Foucault, 2000, p. 170). La genealogía de los saberes descrita en líneas atrás fue la manera como Foucault comprobó la emergencia de la ciencia como un saber disciplinado, pero también surge la disciplinización del saber, es decir, cómo la ciencia se convierte ahora en lo que Foucault denominó policía disciplinaria. Dentro del trabajo policiaco disciplinario del saber se destacan dos aspectos: primero, aparece la universidad, aun cuando desde el medioevo ya funcionaban ciertos espacios para el cultivo del saber. No obstante, Foucault acentúa esta singular aparición debido a la función que ella realiza dentro del disciplinamiento de saberes: seleccionar. Seleccionar los saberes, distribuir su posición, determinar su cantidad y su calidad. Este sería, según Foucault, el papel de la enseñanza. El segundo aspecto que enfatiza el autor es el cambio en la forma del dogmatismo (p. 171). Es decir, se pasa de controlar el contenido de los enunciados a la regularidad de los mismos. En otras palabras, el meollo del asunto reside ya no en lo que se habla sino en quien lo habla. Consiste en una transición de prohibir el saber a disciplinar el saber. Lo que se ha llamado como transición es proporcional a lo que Foucault denominó como desbloqueo epistemológico, un cierto liberalismo disciplinado, regido y enfocado según los estándares de productividad impuestos por los mecanismos mercantiles.

La intervención del Estado en la disciplinización del saber tuvo un trasfondo de poder que confirmaría el saber (¿razón?) de Estado. Sin embargo, hay una salvedad: él solo puede disciplinar los saberes que hacen parte de su historia, más no la historia de los sujetos en lucha (Foucault, 2001, p. 246). Las prácticas disciplinarias no logran permear la conciencia histórica de los sujetos. La gubernamentalidad, en tanto razón de Estado, incorpora una serie de tecnologías de conducción de la conducta propias del pastorado cristiano (Castro-Gómez, 
2013, p. 110 $)^{4}$. La idea es que el Estado ejerza un poder que individualice a través de la implementación de prácticas políticas (Foucault, 1988, p. 9). En esta dirección es que se habla del poder pastoral ejercido mediante la disciplinización del saber. Pero la conciencia de los sujetos no alcanza a ser "disciplinada" u obligada a "obedecer", por lo tanto, se pasa de analizar las posibilidades de control sobre los individuos al poder que se ejerce sobre las poblaciones. Pero más que un poder que se imprime sobre el conglomerado de individuos, se hace un análisis del alcance del poder sobre la vida y las formas de vivir. Las técnicas disciplinarias del poder, que antes se encargaban de vigilar, adiestrar y castigar, son ahora relevadas por nuevas tecnologías de poder sobre la forma en que afectan los procesos del conjunto propio de la vida, como el nacimiento, la muerte, la reproducción, la enfermedad, el trabajo, la educación, entre otras. De este modo, se habla de biopolítica como una tecnología de poder sobre la vida de la población que instala mecanismos de seguridad.

Dentro del conjunto de los procesos que involucran la vida hay uno que se toma, intencionalmente, para lograr el objetivo de esta crítica: la educación, que tomada como biopolítica, avala la idea de considerar la implementación de un dispositivo disciplinario del saber, en este caso como el Icfes. Ahora bien, con base en lo anteriormente expuesto surge la pregunta: ¿cómo y en qué medida se podrá entender que el Icfes, en tanto instrumento interventor del saber, sea comprendido como un dispositivo disciplinario, sobre todo del saber filosófico a nivel de bachillerato en Colombia? Se Ilega a esta comprensión si se revisa el archivo histórico para construir la ruta genealógica del Icfes, sin que sea tomada la genealogía del dispositivo disciplinario con el rigor del caso.

Para comenzar la pesquisa se deben mencionar algunos datos relevantes. Como el hecho de que en 1809 empezaron a realizar una serie de pruebas de admisión a los futuros maestros por parte de la escuela de las Nieves; en 1849, por el Colegio Militar; y en 1867, por la Universidad Nacional ${ }^{5}$, todos ellos en la ciudad de Bogotá. El dilema de las pruebas de Estado durante la primera mitad del siglo XX se relacionaba con el ingreso a la Universidad Nacional para dar cumplimiento

\footnotetext{
${ }^{4}$ En su clase del 8 de febrero de 1978 del curso Seguridad, territorio y población, Foucault, cuando explica la manera en que funciona una tecnología de poder a la que llamó "gubernamentalidad" en la emergencia del Estado moderno, realiza un rastreo a la noción de "gobierno" antes que este adquiera una connotación política. Dicho rastreo lo lleva a señalar que la idea de un gobierno de los hombre hunde sus raíces en el pensamiento pre-cristiano y, posteriormente, cristiano, ya que es allí donde se pone de presente la idea de la organización del poder de manera pastoral y la dirección de las alma (Foucault, 2006, p. 151). De lo anterior se puede inferir que el rasgo pastoral del poder se debe a una influencia semita donde la metáfora del pastor, el rebaño, el redil o la grey empezaron a formar parte del arte de gorbernar. Para analizar un caso donde se evidencie la relación entre poder pastoral y enseñanza de la filosofía, véase: Camelo, D. (2018) P. Andrés Rosa Summa: La enseñanza de la filosofía como subjetivación a mediados del siglo XX. Revista Huila, XVII (69), 75-93.

Estos datos se encuentran en una breve descripción sobre el recorrido histórico de las pruebas de Estado en Rodríguez, D. (2013). Educación Filosófica en Colombia. Módulo de curso electivo. Universidad Nacional de Colombia.
} 
de los decretos 774 de 1912, 571 de 1926, y 1074 de 1934, los cuales ordenaban la presentación de un examen para el ingreso a la educación universitaria. En el año 1942 se estableció como requisito para la obtención del título de bachiller de conformidad con los programas de la enseñanza segunda, determinados por el Ministerio de Educación con previa consulta con la Universidad Nacional. Pero fue hasta en el año 1968 que, mediante el decreto 3156, el Fondo Universitario Nacional creó el Instituto Colombiano para el Fomento de Educación Superior (ICFES). Luego, el presidente de turno López Michelsen publicó el Decreto 089 de 1976 con el cual reestructuró las funciones del Ministerio de Educación, en la que destacaba la de 'auxiliar al Gobierno Nacional en lo relativo a la inspección y vigilancia de la educación superior' y en el que también confirmaba el carácter público del Icfes.

En 1980 se expidió el decreto ley 081 a través del cual se reestructuró el Icfes y se reafirmó su carácter público y adscrito al Ministerio de Educación (MEN), pero siguió manteniendo el oficio de auxiliar al gobierno en el ejercicio constitucional de la educación superior. Más tarde, un año después de la promulgación de la Constitución de 1991, se expidió la Ley 30, por la que "se organiza el servicio público de la educación superior", y reafirmó el sentido público del Icfes, asimismo, su adhesión al MEN. Sin embargo, debido al sinnúmero de cambios en la estructura del poder gubernamental del Estado colombiano, el Icfes fue intervenido en su andamiaje funcional, y se expidió un nuevo decreto, el 1211 de 1993. En 1994 la prueba de Estado estaba compuesta de ocho secciones: dos de actitud (verbal o matemáticas) y seis de conocimientos (Biología, Física, Química, Sociales [geografía, historia y filosofía], Español y Literatura, Matemáticas, y una electiva). Cada una las secciones que conforman la prueba están regidas por los programas analíticos que el Ministerio había establecido en la reforma de 1974, mediante el decreto 080. Seis años después, de nuevo el Icfes fue objeto de modificación y reestructuración mediante el decreto 2662 del 24 de diciembre de 1999, que "establece una estructura horizontal y flexible para la toma de decisiones, con mejores canales de comunicación e interacción, fortaleciendo las áreas misionales" (Icfes, 2003, p. 2).

Desde el año 2000 la resignificación de las Pruebas de Estado conduce a que se tenga un examen basado en competencias como respuesta a las exigencias económicas, políticas y culturales hacia las cuales le apuesta el país. El cambio de enfoque de las pruebas fue una respuesta emergente al contexto de la globalización y los dinamismos innovadores del escenario mundial. Las pruebas empleadas durante los últimos años en el país han correspondido a tres grupos de saberes. Primero, lenguaje e idiomas. Segundo, ciencias sociales, historia, geografía, Filosofía y Matemáticas. Tercero, Biología, química y Física.

No obstante, la Prueba de Estado determinó e influyó, en cierta medida, en la selección de los temas de la enseñanza media, debido al cambio que se produjo 
en lo dispuesto por el decreto 080 de 1974 y 1002 de 1984; el primero señala que la enseñanza de la filosofía debe ser una preparación para la vida y no una simple repetición de conceptos o datos muchas veces históricos y acríticos, mientras que el segundo define cuales deben ser los temas que conecten la enseñanza de la filosofía para la vida y la tradición misma.

Como conclusión se puede sostener que el Icfes es un dispositivo disciplinario en el sentido de que elimina aquellos saberes que son considerados como inútiles a los ojos del modelo económico del Estado, normaliza los saberes y genera así relaciones de poder entre ellos — lo que se ha denominado como alineación de saberes-, asimismo, los jerarquiza de acuerdo a su índole de productividad y de manera que se centraliza su operatividad como dispositivo disciplinario. El Icfes fue la manera como el Estado colombiano intervino en la pugna de los saberes normales y anormales, los productivos e improductivos. No obstante, existe una racionalidad discontinua en la práctica homogeneizante del Icfes y el Ministerio de Educación. La función de inspeccionar y vigilar la enseñanza de los saberes por parte del Icfes provoca una hendidura que permite intuir que hay una distancia entre el uno y otro en lo referente a la homogenización, la cual, se compone de tres elementos: la evaluación como práctica examinadora, el discurso de la transición de ámbitos a componentes y el estándar como técnica normalizadora.

\section{La evaluación como práctica examinadora en Filosofía}

Ha surgido un gran número de aclaraciones acerca de las posibles diferencias entre examen y evaluación en el ámbito educativo. Con gran dificultad, nos lanzaremos a tratar de entablar unas posibles diferencias entre ellas. En Vigilar y castigar Foucault sostiene que el examen involucra las técnicas de jerarquización, que son las encargadas de vigilar, y la sanción, encargada de normalizar. Pues bien, dentro de los dispositivos disciplinarios el examen es de vital importancia ya que mide los vínculos entre las relaciones de poder y las relaciones de saber. Así, el examen utiliza en el interior del dispositivo relaciones de poder que permiten obtener y construir un cierto saber (Foucault, 2002, p. 216). En este sentido, el examen sería una técnica mediante la cual el poder mantiene en línea a aquellos individuos que no se ajustan a la norma del saber. El aprobar o desaprobar el examen sería proporcional a saber qué tan normal se es o no. Para ello, el examen ejercería un poder de individualización disciplinaria haciendo que quien desapruebe se esfuerce por mantenerse en la línea de la norma. El hecho de mantenerlos a todos sobre la línea de la norma disciplinaria del saber mediante el examen es lo que se le denomina como homogenización del saber. Por consiguiente, el examen es una técnica de homogenización que nace de la relación de poder y saber. Ahora, iguarda alguna relación el examen con la evaluación? 

en Colombia: aproximaciones desde Michel Foucault

Es claro que el examen, en cuanto técnica disciplinaria del saber, se circunscribe en el marco de la enseñanza. La escuela, el colegio o la institución educativa es tomada como un dispositivo de escolaridad donde el examen acompaña la acción misma de enseñar, donde mide pero también sanciona — ¿no es acaso la reprobación una sanción epistémica?- - Pero no se limita a ello simplemente. El examen permite generar un vínculo de intercambio de saberes dentro de las relaciones de poder establecidas entre el profesor y el estudiante en el aula de clase. En palabras de Foucault (2002): "La escuela pasa a ser el lugar de elaboración de la pedagogía" (p. 217). En esta dirección, se ajustan las palabras de Flórez (1999) al sostener que:

La evaluación de la enseñanza no es una actividad externa a la enseñanza misma, sino que es parte y condición endógena del proceso mediante el cual el estado interior del aprendiz se mueve, se confronta, se desequilibra y se re-estructura frente a lo desconocido que indaga. [...] Se puede decir que enseñar es evaluar, retroalimentar, reconstruir la pregunta cada vez que la mente del alumno se moviliza en su búsqueda y se compromete con una respuesta que puede ser o no ser al mismo tiempo. (p. 20)

Si se considera un poco la legislación educativa en materia de evaluación, vale traer a colación el decreto 230 del 11 de febrero de 2002 sobre la evaluación de los aprendizajes en el cual se define la evaluación como una acción permanente e integral, es decir, un proceso continuo. En el caso de la filosofía debe entenderse en el sentido de que la evaluación es una práctica que examina el aprendizaje de los saberes en los estudiantes mediante la relación de poder que estos interactúan con el profesor en un espacio concreto que es el aula de clase. Esto demuestra la razón por la que ha sido objeto de tantas modificaciones a lo largo de los últimos treinta años. De hecho, los decretos 080 y el 1002 fueron evidencia de que la evaluación de la filosofía mostrara que su enseñanza no estaba desencarnada de la vida cotidiana, que no consistía en la memorización de nociones o autores. La evaluación en la enseñanza de la filosofía a nivel de bachillerato es y ha sido resultado de un proceso de jerarquización y normalización en el que el estudiante debía ser el que confirmara que dichos cambios sí eran los efectivos.

Los procesos individualizantes como la memorización, la deducción, el análisis, entre otros, no deben ser tomados como el todo en la evaluación de los aprendizajes, como tampoco se debe caer en el otro extremo de tomar los contendidos como el fin último de la evaluación. La evaluación, como es un proceso permanente, continuo e integral, ha de llevar a la confrontación con el saber, tomarlo, sacarlo, botarlo, volverlo a recoger a fin de que el estudiante sienta que lo puede manejar. Solo así se podrá pensar si la técnica examinadora de la evaluación habría cumplido su cometido. 


\section{Cambio de discurso: de ámbitos a componentes de la Filosofía}

En La arqueología del saber Foucault (1979) sostiene que los discursos no se deben tomar como una totalidad vista desde afuera, sino que existe la necesidad de partir de las unidades que los componen con el fin de definir unas reglas que permitan construir unos cánones que posibiliten erigir eventualmente otros enunciados aparte de estos (p. 43). El tránsito de un discurso está condicionado por las reglas emergentes de las unidades que lo conforman, es decir, las mismas que lo individualizan. Ahora bien, ¿qué reglas fueron las que obligaron a dejar el discurso sobre los ámbitos para comenzar a hablar de componentes en la enseñanza de la filosofía a nivel de bachillerato en Colombia?

Amparada por las disposiciones definidas en la Ley general de educación en 1994, se prolifera la urgencia de evaluar por competencias como una muestra de apertura al mundo moderno. Por su parte, ya el Ministerio había sembrado dicha necesidad. Ahora le correspondería al Icfes preocuparse, en su función de panóptico del saber, por agrupar en las preguntas de las pruebas de Estado tanto la teoría como la práctica del aprendizaje, al igual que el procedimiento, y averiguar que el estudiante tenga una aproximación más crítica frente a lo que se dispone, buscando que la prueba abandone su rol de medición y pase a ser también formación. Esta es la razón por la cual la evaluación de la filosofía está conformada por ámbitos —aspecto teórico - y competencias —aspecto práctico- (MEN, 2004, p. 189).

A principios de la década del 2000, la prueba estaba constituida por cinco ámbitos que son los que corresponden al grupo temático a evaluar. Serían algo así como la norma epistémica de la enseñanza del saber. Dichos ámbitos fueron especificados por el Icfes de la siguiente manera: la epistemología, la antropología, la ética, la estética y la ontología. A continuación, se hará una breve descripción a partir de la presentación hecha por Ortiz \& Verano (2001) en el texto La filosofía: Entre el conocimiento de la tradición filosófica y el reconocimiento de la información. El primero de ellos es el ámbito epistemológico en el cual "se evalúan los grandes interrogantes en torno al conocimiento y sobre las interpretaciones y respuestas que éstos han dado los filósofos más destacados a través de la historia de la filosofía" (Ortiz \& Verano, 2001, p. 4). El segundo, el ámbito antropológico, "comprende la reflexión sobre el hombre en su relación con el mundo de la naturaleza, con la sociedad y consigo mismo" (p. 4). El tercer ámbito es el ético. En él se "incluye la reflexión filosófica sobre el conjunto de principios, normas y valores que fundamentan las acciones de los grupos humanos en las distintas épocas de la historia" (p. 4). El cuarto es el ámbito estético, en el que "se aborda la interpretación filosófica de los problemas y concepciones elaboradas sobre creaciones artísticas como la obra poética, pictórica o musical" (p. 4). El quinto y último ámbito es el ontológico. En él se formulan las preguntas que tratan las 
problemáticas relacionadas con el sentido y la constitución del ser, con aquello que define el ser de las cosas, la realidad, el mundo (Ortiz \& Verano, 2001, p. 4).

Sin embargo, en 2006 la estructura de la prueba cambia rotundamente al pasar del discurso de los ámbitos al de los componentes. Este cambio, según el documento oficial del Icfes, se debió al indagar sobre la

relevancia de algunos de los componentes (ámbitos) en la prueba, teniendo en cuenta que debido a la intensidad horaria mínima que tiene la asignatura en la mayoría de instituciones y a que su enseñanza se imparte a partir de décimo grado, es muy difícil que la población estudiantil pueda tener acceso suficiente a algunos de ellos. (Melo, 2007, p. 14)

Por ello, la evaluación de la filosofía busca dar espacios al estudiante "frente a un caso concreto, aplicando lo aprendido" (Melo, 2007, p. 190). El Icfes lo que escudriñó fue que los ámbitos tuvieran algunos rasgos que no fueran ajenos entre sí, sino que tuvieran ciertas características transversales entre ellos. Esta transversalidad fue lo que permitió reformular la evaluación en filosofía al pasar de los cinco ámbitos a tres componentes que, según el criterio del Icfes, son los problemas más propios de la filosofía. Según la justificación que presenta la entidad, no se trata de un reduccionismo de los componentes, sino de una jerarquización de los saberes.

A continuación, se presentan los que serían los nuevos componentes de la evaluación de filosofía de acuerdo con la descripción dada por el documento del Icfes titulado Examen de Estado: La enseñanza y la evaluación en filosofía del año 2005:

La pregunta del hombre frente a su mundo social y cultural: Implica el cuestionamiento del hombre por su relación con las manifestaciones histórico-culturales y ético-políticas. En este componente se puede indagar por distintas relaciones del hombre con la cultura, como pueden ser el arte, las pautas morales, el lenguaje y las connotaciones que estos temas tuvieron en las distintas matrices culturales. Así mismo, pueden realizarse preguntas que giren en torno a teorías del poder actuales o pasadas y sus implicaciones en la vida del estudiante y en la sociedad colombiana.

La pregunta por el ser: Este es un problema clásico y propio de la filosofía, por lo tanto, se mantienen sus implicaciones, especificándolo en la pregunta por la relación entre el ser, el mundo y el hombre, sin entrar en la discusión terminológica entre metafísica y ontología, sino ahondando en la relación entre el hombre y la totalidad, las preguntas que este encuentro genera y las posiciones que desde la filosofía se han producido.

La pregunta por el conocimiento: Incluye cuestionamientos acerca del problema del conocimiento en general, de la ciencia y de la influencia de ésta en la constitución del hombre y de la imagen que este se forma de la realidad, así como sobre la influencia de esta imagen en la constitución 
de la ciencia y del conocimiento en la actualidad. Por lo tanto, en este componente pueden encontrarse preguntas que giren en torno a la reflexión filosófica que genera la ciencia en el hombre y en la sociedad, así como una reflexión epistemológica sobre la misma ciencia [cursiva añadida]. (Icfes, 2005, p. 13)

Así las cosas, el paso de ámbitos a componentes, ¿acaso representó un cambio de discurso o un discurso de cambio? Lo cierto es que de este discurso de transición se pueden concluir dos ideas. La primera es que no cabe duda que este gesto constituye un intento por disminuir la brecha entre la enseñanza de la filosofía y el contexto donde interactúa. Acercar el saber filosófico a las circunstancias en las que se desenvuelve el estudiante en tanto sujeto condicionado por las estructuras fue el principal motivo de este cambio, o sea, de esta transición. Lo que se pretende, por consiguiente, es consolidar un vínculo comunicativo entre el saber disciplinar y el contexto, y este vínculo sería, desde luego, el saber filosófico. En segunda instancia, la evaluación de la filosofía debe ir tras la formación integral del sujeto. Más allá de las técnicas de evaluación, la preocupación ha de gravitar en torno a establecer relaciones entre el saber y el contexto, la manera de cómo el saber filosófico no se vea desencarnado de la realidad, sino que, por el contrario, pueda vincularse de tal modo que el estudiante confirme que lo que aprende es lo que necesita para vivir.

\section{La educación por competencias como Tecnología de poder en Filosofía}

Como tercer elemento que compone esta crítica a las prácticas de enseñanza de la Filosofía se encuentra la educación por competencias interpretándola como tecnología de poder. Para ello, se recordará ligeramente qué entendía Foucault por tecnología y cuáles son sus atributos más característicos. El uso de la noción "tecnología" es bastante compleja si se tienen en cuenta las estimaciones hechas por Castro-Gómez (2013) quien sostiene la evidencia de una continua imprecisión del término empleado por Foucault (p. 35). Pero para ser más concretos se ha de definir tecnología como el modo estratégico a través del cual una práctica se desenvuelve dentro del entramado de poder. En otras palabras, son los medios por los que una acción logra eficazmente llegar a su objetivo trazado. Pero no debe ser tomado como mero utensilio conceptual manejado por uno o unos sujetos. De ahí que tecnología no es un instrumento, sino como se refería Foucault cuando hablaba de la tecnología del yo, un modo en que un individuo actúa sobre sí mismo. Tecnología, entonces, es un modo (Foucault, 1990, p. 49). Ahora bien, para referirnos a tecnología de poder debemos recordar la clase del 18 de enero de 1978 del curso Seguridad, territorio y población donde Foucault enmarca dicho concepto bajo los términos de dispositivos de seguridad. 

en Colombia: aproximaciones desde Michel Foucault

Dentro de toda su genealogía del poder, Foucault lo que identifica es la transición de unas prácticas disciplinarias que en la época clásica se centraban en el castigo y que poco a poco se fue pasando a unas prácticas cuyas racionalidades estuvieran fundadas en la seguridad. En este sentido, la conducta de los individuos pasaría de ser coartada y castigada a ser regulada y vigilada. Por lo tanto, el poder en esta trama de relaciones debe verse como una acción que regule, pero desde la libertad y no desde la coerción. En la base de esta regulación se encuentra lo que Foucault llamó "procedimientos de normalización" (Foucault, 2006, p. 71). Pero el mencionado procedimiento demanda una aclaración pertinente para lo que se pretende en esta parte de la crítica.

La tecnología de poder a la que nos referimos es al poder disciplinario de normación correspondiente a la seguridad y no al castigo ${ }^{6}$. Esto quiere decir que la tecnología en mención busca establecer un estereotipo de comportamiento sobre el cuerpo a través de técnicas de adiestramiento de acuerdo con la norma establecida. A partir de aquella norma se define si el comportamiento de un sujeto es normal o no, si es apto o no. Por esta razón es que Foucault prefiere decir, cuando se refiere a la diferencia entre lo normal y lo anormal, que las técnicas disciplinarias se tratan más por normación que por normalización, lo que resalta el carácter primario y fundamental de la norma (Foucault, 2006, p. 76). Sin embargo, los dispositivos de seguridad no están dirigidos a los individuos sino a la población, a la masa. Su punto de partida, además, son los hechos, es decir, los acontecimientos a partir de los cuales se puedan dictaminar ya no en un sentido de normalidad o anormalidad, sino en un sentido de peligrosidad (CastroGómez, 2013, p. 79). En esta dirección, todo aquello que atente o ponga en riesgo el equilibrio del Estado en su fase "normal" será calibrado como peligroso y dicha peligrosidad será proporcional a su nivel de amenaza. Ello conllevaría la implementación de diferentes estrategias para que el Estado mantenga su normalidad, una de ellas es la educación, entendida como una estrategia de estatalización que el Estado implemente para la seguridad de la población.

Pero no es la educación en sí misma la que asegura dicha estrategia, es la educación en relación con otras, tanto internas como externas, las que posibilitan la estrategia. En otras palabras, la educación junto con la economía, la política, las religiones, entre otras, conforman un entramado estratégico de poder estatal, pero a su vez dichas estrategias involucran ciertos discursos que soportan dicho entramado. En este caso, dentro de la estrategia de educación se resalta de manera especial el discurso sobre las competencias.

${ }^{6}$ Sería oportuno aclarar que los dispositivos disciplinarios son los que actúan por normalización, pero las técnicas disciplinarias lo hacen por normación. 
Así pues, se identifica un número importante de autores de cuyas teorías se han nutrido para lograr su formulación. A partir de las contribuciones de pensadores como Noam Chomsky y Vygotsky, entre otros, el discurso de la educación por competencias se logró introducir en la práctica del quehacer educativo. Sin embargo, una exposición clara como la que presenta G. Ryle (2005) en el texto El concepto de lo mental permite tener una consideración que se ajusta a nuestra intensión. En el segundo capítulo de la obra en mención titulada Saber qué, saber hacer, Ryle pretende exponer la tesis según la cual las expresiones mentales son, en sí mismas, expresiones autónomas.

Ryle (2005) comienza cuestionando si las categorías tradicionales de inteligente o tonto que le son atribuidas a una persona son proporcionales entre sí. Tal parece que esta dicotomía rompe con lo que el autor ha denominado leyenda intelectualista (p. 46). Para Ryle es importante aclarar que ser inteligente es una cosa y tener conocimiento es otra, debido a que se consideran las operaciones intelectuales como el centro de la conducta mental y cuya actividad fundamental consiste en responder a problemas. Por otro lado, cuando el autor habla de intelecto lo hace entendiéndolo como la clase especial de operaciones que constituye el teorizar. El intelecto estaría al servicio del conocimiento (Ryle, 2005, p. 40). Por consiguiente, esta sería la diferencia entre las operaciones intelectuales y las cualidades mentales. Así, ser inteligente no es siempre expresión ni comprobación de una teoría. Es más, construir una teoría es ya una práctica inteligente.

Ser "inteligente" o "ignorante" no son categorías atribuibles al conocimiento sino a la habilidad o ausencia de habilidad para hacer cierto tipo de cosas (Ryle, 2005, p. 42). Por tanto, saber hacer y saber qué son dos aspectos yuxtapuestos entre sí donde aprender a hacer un oficio es un extremo y saber algún conocimiento es otro. Esto iría a contrapelo de lo que ha sostenido la leyenda intelectualista que cree que "toda operación considerada inteligente debe ser precedida por un reconocimiento intelectual de reglas y criterios" (Ryle, 2005, p. 43), es decir, que la teoría antecede a la práctica. Es a esta concepción a la que pretende atacar Ryle cuando arguye que es falsa, y sostiene que una acción inteligente no implica una ejecución de una doble acción de considerar y ejecutar, es decir, que una acción eficiente no siempre es producto de una eficiente teoría. En Palabras del autor, esta misma idea se expresa así: "Es posible llevar a cabo inteligentemente ciertas operaciones sin que sea posible tener en cuenta las proposiciones que determinan cómo ejecutarlas. Algunas acciones inteligentes no están reguladas por el conocimiento previo de los principios que la rigen" (Ryle, 2005, p. 44).

Con esto, Ryle sostiene que la categoría que le es aplicada a las prácticas inteligentes no puede definirse en términos intelectuales, pues pensar en lo que se está haciendo no connota pensar en lo que se debe hacer y hacerlo efectivamente. De hecho, Ryle (2005) señala la ineludible distinción entre los hábitos y aptitudes inteligentes. Los hábitos, afirma, se crean por mera rutina, y esta, a su vez, con 

en Colombia: aproximaciones desde Michel Foucault

repeticiones; las aptitudes inteligentes, mediante el adiestramiento (p. 57). Afirma también que gran parte del aprendizaje se da es por rutina al punto de que la conceptualización y la habilidad cuantitativa se desarrolla por estímulos automáticos, pero el adiestramiento implica un estímulo mediante la crítica y el ejemplo. En palabras del autor: "Se trata de aprender a hacer cosas pensando en lo que se está haciendo, de modo que cada acto ejecutado es en sí mismo una nueva lección que ayuda a mejorar la acción" (Ryle, 2005, p. 57).

Ahora bien, ¿es el adiestramiento, como lo llama Ryle, la práctica de educar competentemente a un sujeto? Para responder a esta inquietud se ha de recurrir a la definición de competencias manejada desde la institucionalidad oficial. De acuerdo con el documento Sistema nacional de evaluación estandarizada de educación (2013), se entiende por competencias al:

[...] conjunto de conocimientos, habilidades, actitudes, comprensiones y disposiciones cognitivas, meta-cognitivas, socio-afectivas, comunicativas y psicomotoras apropiadamente relacionadas entre sí para facilitar el desempeño flexible, eficaz y con sentido de una actividad o de cierto tipo de tareas en contextos relativamente nuevos y retadores. (Icfes, 2013, p. 10)

Dicho esto, el discurso de las competencias aplicado a la educación consistiría en la transmisión de conocimientos demostrativos ${ }^{7}$. De este modo, la educación por competencias consistiría entonces en desarrollar un "saber-hacer en contexto" (p. 10). En consecuencia, es competente aquel que utiliza los conocimientos que posee y los que en un futuro poseerá con el fin de enfrentar los diferentes problemas o situaciones que se presentan. Con base en lo afirmado anteriormente, es viable preguntarnos: ¿Cómo empalmar el discurso de la educación por competencias en la enseñanza de la filosofía a nivel de bachillerato?

En atención a lo establecido en el documento oficial del Ministerio de Educación Nacional (MEN) Orientaciones pedagógicas para la filosofía en la educación media (2010) se definen tres competencias para el ejercicio del filosofar en el marco de la educación media en Colombia. Según el documento citado, la filosofía es un saber específico que requiere del desarrollo de unas competencias propias que estén asociadas al pensamiento crítico, a la comunicación y a la creatividad (pp. 31-32). En efecto, por normatividad, las competencias establecidas por el MEN son: la competencia crítica, que es aquella que le permite al sujeto emanciparse de las opiniones (doxa) y le permite apreciar la parcialidad de las pretensiones de verdad y validez (episteme). La competencia dialógica, como la práctica comunicativa donde la percepción y el análisis se combinan para mostrar cómo la interpretación no es una labor pasiva, sino un acto creativo; y, por último, la

El documento original habla de conocimientos declarativos. No obstante, se considera — si se parafrasea a Ryle - que es más preciso hablar de conocimientos demostrativos en el sentido de que se abre la posibilidad de demostrar con el saber-hacer un saber-qué. 
competencia creativa, relacionada con el pensamiento formal, es decir, con el manejo de conceptos, operaciones y principios lógicos (MEN, 2010, pp. 32-33).

Pues bien, estas tres competencias, de las que se dice son las propias de la filosofía, parecen no encajar con las mencionadas y establecidas por el dispositivo disciplinario del saber que es el Icfes, en su práctica examinadora que resulta siendo la prueba de Estado — hoy llamada Prueba Saber $11^{\circ}$ —, ya que define tres competencias diferentes en sus instructivos canónicos. A las que se hace referencia son: competencia interpretativa, que involucra acciones como el reconocimiento de las tesis principales en los textos filosóficos, la deducción de las consecuencias y las implicaciones de los problemas; la competencia argumentativa, cuyas acciones comprende la identificación de los argumentos de los autores frente a los problemas filosóficos presentes en los textos examinados y producidos a lo largo de la historia; y la competencia propositiva, que consiste en el manejo de conceptos y en la resolución de confrontaciones de tesis entre distintos autores de una misma o de diversas épocas, asimismo, el establecimiento de relaciones y diferencias entre esos conceptos y las posiciones científicas, religiosas y artísticas, entre otras (Icfes, 2011, p. 21).

De esta manera, se demuestra que en el discurso de la educación por competencias en la enseñanza de la filosofía a nivel de bachillerato en Colombia se presenta una gran incongruencia entre los propósitos que establece el MEN, en términos de aquello que debe ser evaluado, y el Icfes, en la función técnica de diseñar, aplicar y analizar las evaluaciones. En resumen, la tesis que se ha dispuesto a demostrar es esta: la educación por competencias en el marco de la enseñanza de la filosofía en Colombia es una tecnología de poder en cuanto que es un modo de ejercer un saber dentro de unas relaciones didácticas cuya racionalidad se encuentra fundamentada en un discurso discontinuo entre la norma emanada del MEN y el instrumento examinador propuesto por el Icfes.

\section{Referencias}

Castro-Gómez, S. (2013). Historia de la gubernamentalidad: Razón de Estado, liberalismo y neoliberalismo en Michel Foucault. Siglo del Hombre Editores.

Flórez, R. (1999). Evaluación pedagógica y cognición. McGrawHill.

Foucault, M. (1979). Arqueología del Saber. (A. Garzón del Camino, trad.). Siglo XXI Editores.

Foucault, M. (1985). ¿Qué es la Ilustración? En Saber y verdad (pp. 197-207). La Piqueta. 

en Colombia: aproximaciones desde Michel Foucault

Foucault, M. (1990). Tecnologías del Yo. (M. Allendesalazar, trad.). Paidós.

Foucault, M. (2000). Defender la sociedad. Curso en el Collége de France (19751976). Fondo Cultura Económica.

Foucault, M. (2001). El sujeto y el poder. En H, Dreyfus \& P. Rabinow. (Eds.), Michel Foucault: más allá de estructuralismo y la hermanéutica (R. Paredes, trad.). (pp. 241-259). Nueva Visión.

Foucault, M. (2002). Vigilar y castigar. Siglo XXI Editores.

Foucault, M. (2003). ¿Qué es la Crítica? En Sobre la Ilustración. (J. De la Higuera, E. Bello \& A. Campillo, trads.). Técnos.

Foucault, M. (2006). Seguridad, Territorio, Población. Curso en el Collége de France (1977-1978). (H. Pons, trad.). Fondo de Cultura Económica.

Foucault, M. (2009). Un lectura de Kant. Introducción a la Antropología en sentido pragmático. Siglo XXI Editores.

Icfes (2003). Transformación del Icfes. [Documento técnico]. Icfes.

Icfes (2004). Evaluación por competencias. Matemáticas, Ciencias Sociales y Filosofía. Evolución de las Pruebas de Estado. Cooperativa Editorial Magisterio.

Icfes (2005). Examen de estado: La enseñanza y la evaluación en filosofía. Icfes.

Icfes (2011). Orientaciones para el examen de Estado de la Educación Media. Icfes saber $11^{\circ}$. Icfes.

Icfes (2013). Sistema Nacional de evaluación estandarizada de la educación. Alineación del examen Saber $11^{\circ}$. Icfes.

Melo, C. S. (2007). Fundamentación conceptual del área de Filosofía. Icfes.

Ortiz, G. \& Verano, L. (2001). La filosofía: entre el conocimiento de la tradicón filosófica y el reconocimiento de la información. Icfes.

Rodríguez, D. (2013). Educación Filosófica en Colombia. Módulo de curso electivo. Bogotá. Universidad Nacional de Colombia.

Ryle, G. (2005). El concepto de lo mental. Paidós. 